\title{
Non incappiamo in errori nella grafia di capasanta
}

\author{
Sara Giovine
}

PUBBLICATO: 16 MARZO 2021

\section{Quesito:}

Diversi lettori si sono rivolti al servizio di consulenza linguistica per sapere quale sia la grafia più corretta tra capasanta, capa santa, cappasanta o cappa santa. Ci viene inoltre chiesto quale sia il plurale della parola.

\section{Non incappiamo in errori nella grafia di capasanta}

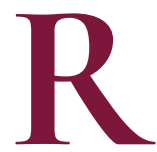

ispondiamo subito ai nostri lettori che tutte le varianti citate risultano ammissibili: la grafia più diffusa, per le ragioni storiche ed etimologiche che si diranno, è infatti capasanta, ma la voce, come indicato nei principali dizionari dell'uso, può essere scritta anche in forma non univerbata, ossia con grafia analitica (capa santa), o nella variante italianizzata cappasanta (ugualmente ammessa anche nella forma analitica cappa santa).

La grafia capasanta, con consonante scempia, si spiega con l'origine settentrionale del termine: si tratta infatti di una forma regionale veneta (divenuta poi di uso corrente anche in italiano), impiegata come nome comune di un mollusco commestibile con conchiglia a due valve, della famiglia dei Pettinidi, il cui nome scientifico è Pecten jacobaeus. Il termine è composto dal sostantivo capa, variante appunto veneta, con scempiamento della consonante, di cappa ('nome comune di varie specie di molluschi marini bivalvi'), e dall'aggettivo santa.

Il primo elemento del composto, come chiarito dai dizionari etimologici, deriva dal sostantivo cappa nel suo significato primario di 'mantello con cappuccio', attraverso un passaggio semantico ricostruito da Folena 1954, che lo riconduce alla somiglianza tra la forma della conchiglia e quella dell'indumento: secondo lo studioso, tale accostamento nascerebbe in particolare da una "metafora visiva applicata alla conchiglia con le sue scanalature e ondulazioni, simili alle pieghe verticali a raggiera di un mantello". La forma, attestata dalla metà del Quattrocento (come ci documenta sempre Folena 1954) e diffusa soprattutto in area settentrionale, in cui risulta prevalente la grafia con consonante scempia capa, è stata variamente impiegata nei secoli successivi per indicare, da sola o piu spesso accompagnata da aggettivi o altre specificazioni, diverse tipologie di pesci o molluschi (elencati tra gli altri da Cortelazzo 2007), tra cui la capa da deo o cappa di deo 'coltellaccio (Solen vagina)', la capa longa o cappa lunga 'cannolicchio (Solen siliqua o Solen vagina)', la capa tonda 'tellina (Cardium edule o Cardium clodiense)' e appunto la capa santa.

Quanto al secondo elemento del composto, il ricorso all'aggettivo santa per qualificare tale specifica varietà di mollusco si spiega invece con ragioni di carattere storico, brevemente illustrate anche da alcuni dizionari sincronici (tra cui il GRADIT e il Devoto-Oli): la conchiglia del mollusco era infatti usata come contrassegno dai pellegrini che si recavano al santuario di San Giacomo a Santiago de Compostela, in Spagna. Questi, dopo aver usato la valva inferiore della conchiglia per bere durante il pellegrinaggio, la riportavano a casa come ricordo del viaggio: in breve tempo la conchiglia è così divenuta simbolo del pellegrinaggio, spesso cucita sugli abiti o sul cappello dai pellegrini (cfr. Perusini I968-70, pp. 290-9I), motivo per cui la capasanta è nota anche con il nome di conchiglia dei pellegrini o conchiglia di San Giacomo (e a quest'ultima denominazione è da ricondurre anche il nome scientifico 
del mollusco, Pecten jacobaeus 'pettine di Giacomo').

Come anticipato, le origini del composto sono quindi da ricercare in area settentrionale, e in particolare nel veneziano, in cui il sostantivo capa è, non a caso, diffusissimo come "termine collettivo [...] dato da' pescatori a molte differenti sorte di conchiglie delle nostre marittime località" (cfr. Boerio): secondo Cortelazzo 2007, il primo a impiegare la forma capa santa è lo storico veneziano Marin Sanudo, in un testo della fine della Quattrocento dedicato all'origine e alla storia della città lagunare, descritta nel dettaglio dal punto di vista delle strutture amministrative, della società, del costume e dell'economia (incluso il redditizio settore della pesca):

Queste sono le sorti de pessi vendono freschi in Venetia: [...] cape longhe, cape penevaze [= peveraze 'vongole (Venus gallina)'], cape sante, cape a deo, cape pisote, cape da canal, cape. (M. Sanudo, De origine, situ et magistratibus urbis venetae ovvero La cittá di Venetia, I493)

Dal veneziano, il termine si sarebbe poi progressivamente diffuso non solo nel resto dell'area veneta, ma anche in altre varietà settentrionali, tra cui il ligure, il friulano, il triestino, il romagnolo, per estendersi infine, seguendo la costa adriatica, anche al marchigiano, all'abruzzese e quindi al napoletano (cfr. LEI XI, 427-28). Come è avvenuto per molte altre voci regionali afferenti al campo semantico del cibo e della cucina, poi entrate a far parte del comune lessico italiano (come pizza o mozzarella, per citare solo due tra gli esempi più noti), a partire da metà Ottocento l'uso di capasanta ha cominciato a estendersi all'intera penisola, fino a divenire del tutto comune anche in italiano, sia nella variante regionale con consonante scempia, sia in quella con pronuncia e grafia italianizzata cappasanta. Secondo il GRADIT, la prima attestazione italiana del termine risalirebbe al I858, nello scrittore veneto Ippolito Nievo, ma, come indicato nel LEI, la voce è in verità già accolta, circa un decennio prima, nel Vocabolario universale della lingua italiana (i847) e ancor prima, alla fine del Settecento, nella raccolta lessicale di storia naturale del lessicografo tedesco Philipp Nemnich, che la include (nelle due varianti capa santa e cappa santa) nella sezione riservata all'italiano.

Nell'italiano contemporaneo, in cui la forma risulta ormai del tutto acclimatata, risultano quindi ammesse entrambe le varianti grafiche del composto, quella riconducibile alle origini regionali della forma (capasanta) e quella adattata alla pronuncia italiana (cappasanta), sebbene quest'ultima risulti decisamente minoritaria nell'uso rispetto alla prima (28.500 risultati di cappasanta nelle pagine italiane di Google il I8/II/2020 contro i ir2.ooo di capasanta). Ugualmente ammesse anche le corrispondenti varianti analitiche, con mancata fusione grafica dei due elementi del composto, che sono però anch'esse nettamente minoritarie (II.900 occorrenze in rete di capa santa e 7.IOo di cappa santa), probabilmente in virtù del fatto che il composto, attestato da tempo nella nostra lingua, risulta ormai percepito come una parola unitaria.

Infine, per quanto riguarda la formazione del plurale del termine, essa prevede la declinazione di entrambi i costituenti del composto (e dunque la capasanta > le capesante): la forma appartiene infatti alla categoria dei composti Nome + Aggettivo, che, come indicato nelle principali grammatiche dell'uso (tra cui Serianni i989, III I40), formano il proprio plurale appunto modificando le desinenze di entrambi gli elementi componenti, come la cassaforte > le casseforti, la terracotta > le terrecotte.

\section{Nota bibliografica:}

- Giuseppe Boerio, Dizionario del dialetto veneziano, Firenze, Giunti, 2006 [ripr. facs. dell'ed. Venezia, Premiata tipografia di Giovanni Cecchini, I856]. 
- Manlio Cortelazzo, Dizionario veneziano della lingua e della cultura popolare nel XVI secolo, Limena, La Linea, 2007.

- Gianfranco Folena, Cappe e capparazze, in "Lingua nostra", XV (I954), p. 75.

- Philipp Andreas Nemnich, Allgemeines Polyglotten-Lexicon der Naturgeschichte mit erklärenden Anmerkungen, 4 voll., Hamburg-Halle-Leipzig, Adam Friedrich Böhme-Conrad Müller, I793-I798.

- Gaetano Perusini, Amuleti ittici, in "Bollettino dell'Atlante linguistico mediterraneo", IO-I2 (I968I970), pp. 283-306.

- Adriana Rossi, I nomi dei pesci, in "Studi di lessicografia italiana", VI (I984), pp. 67-232.

- Vocabolario universale della lingua italiana. Edizione eseguita su quella del Tramater di Napoli con giunte e correzioni, 8 voll., Mantova, F.lli Negretti, I845-1856.

\section{Cita come:}

Sara Giovine, Non incappiamo in errori nella grafia dicapasanta, "Italiano digitale", 2021, XVI, 2021/1 (gennaio-marzo)

DOI: $10.35948 / 2532-9006 / 2021.5491$

\section{Copyright 2021 Accademia della Crusca}

Pubblicato con licenza creative commons CC BY-NC-ND 